\title{
Role of Lung Scan for Detection of Pneumothorax in Operation Theatre
}

\author{
Poudel SC, ${ }^{1^{*}}$ Rayamajhi AJ, ${ }^{1}$ Bhattarai $P R,{ }^{1}$ Yadav $R K^{1}$
}

\begin{abstract}
Affiliation:
1. Department of Anesthesiology, Civil Service Hospital, Kathmandu, Nepal.
\end{abstract}

\section{ARTICLE INFO}

\section{Article History}

Received : 06 Oct, 2016

Accepted : 21 Oct, 2016

Published : $20 \mathrm{Dec}, 2016$

C A Authors retain copyright and grant the journal right of first publication with the work simultaneously licensed under Creative Commons Attribution License CC - BY 4.0 that allows others to share the work with an acknowledgement of the work's authorship and initial publication in this journal.

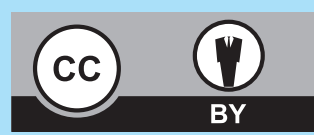

\section{* Corresponding Author}

Dr. Subash Chandra Poudel

$$
\text { Consultant }
$$

Department of Anesthesiology

Civil Service Hospital, Kathmandu, Nepal

Email: subashchandrapaudel@gmail.com

\section{Citation}

Poudel SC, Rayamajhi AJ, Bhattarai PR, Yadav RK. Role of Lung Scan for Detection of Pneumothorax in Operation Theatre. BJHS 2016; 1 (1) 1: 75-77.

\section{ABSTRACT}

latrogenic pneumothorax is life threatening complication that an anesthesiologist may encounter in operating room while performing various procedure such as central venous catheterization brachial plexus block paravertebral block or during surgery like pyelolithotomy, laparoscopic cholecystectomy, percutaneous nephrolithotomy. Ultrasound can be the diagnostic tool for prompt diagnosis and managementof these situations. As there is overwhelm use of ultrasound in emergency department and ICU setting, by understanding few ultrasonic terms like lines, modes, signs and points of lung scan it can be easily utilized in operation room. Likewise, lung ultrasounds in emergency (BLUE) and fluid administration by lung ultrasound (FALL) are recently being recommending to be used in emergency and ICU. We report two cases where lung scan was beneficial for management of pneumothorax in our operation theater.

\section{KEYWORDS}

Lung scan, pneumothorax, PCNL 


\section{INTRODUCTION}

The role of anesthesiologist has been extended as perioperative sonologist as overwhelm use of ultrasound in operation theatre. latrogenic pneumothorax caused by various anaesthetic procedures like centra venous catheterization, brachial plexus block or paravetebral blocks and various surgical procedures like percutaneous nephrolithotomy, pyelolithotomy, and laparoscopic cholecystectomy can be detected early on operation table using lung scan. ${ }^{1}$ Pneumothorax is a medical emergency that needs early detection and intervention. Lung ultrasound avoids need of chest X-ray and risk of transports of patient to radiological suite.

Here we report two cases where we used lung ultrasound to diagnose or rule out pneumothorax intra-operatively.

\section{CASE REPORTS}

\section{Case1}

Fifty-five years old female, ASA 1, diagnosed as left upper pole renal calculi was scheduled for percutaneous nephrolithotomy. Pre-anesthetic check up was done, written consent was taken and she was premeditated with diazepam $5 \mathrm{mg}$ day prior to surgery. On day of surgery standard monitoring ECG, SPO2, BP was attached with intravenous line secured on left dorsum of hand. General anesthesia was induced with $2 \mathrm{mg}$ midazolam, 125 micrograms of fentanyl $100 \mathrm{mg}$ propofol and $6 \mathrm{mg}$ vecuronium and trachea was intubated. Patient was kept in prone position during surgery. Anesthesia was maintained with isofurane, vecuronium and fentanyl. During surgery, breath holding was done at the end of inspiration and infra costal superior calyceal puncture was done as the part of surgical procedure. Then surgery was carried on and was uneventful. Muscle relaxant was reversed wit $2.5 \mathrm{mg}$ neostigmine and $0.6 \mathrm{mg}$ glycopyrolate and trachea was extubated. But immediately after extubation spO2 decreased up to $85 \%$ gradually, then patient was ventilated manually and lung scan (My Sono, Medison) was done using linear probe as there was decreased breathe sound during auscultation on left side. The scan showed absence A-line (figure 1), lung sliding in B mode, Barcode sign in $M$ mode (figure 2) and lung point on both $B$ and $M$ mode confirming pneumothorax. Thus, after placement of chest tube oxygen saturation was maintained and patient shifted to recovery room.
Figure 1:

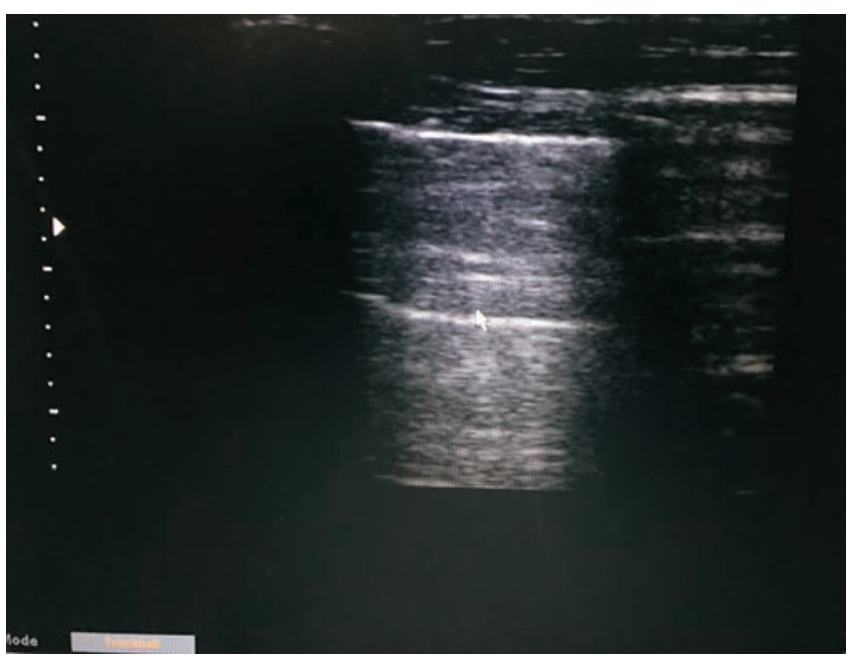

Figure 2 :

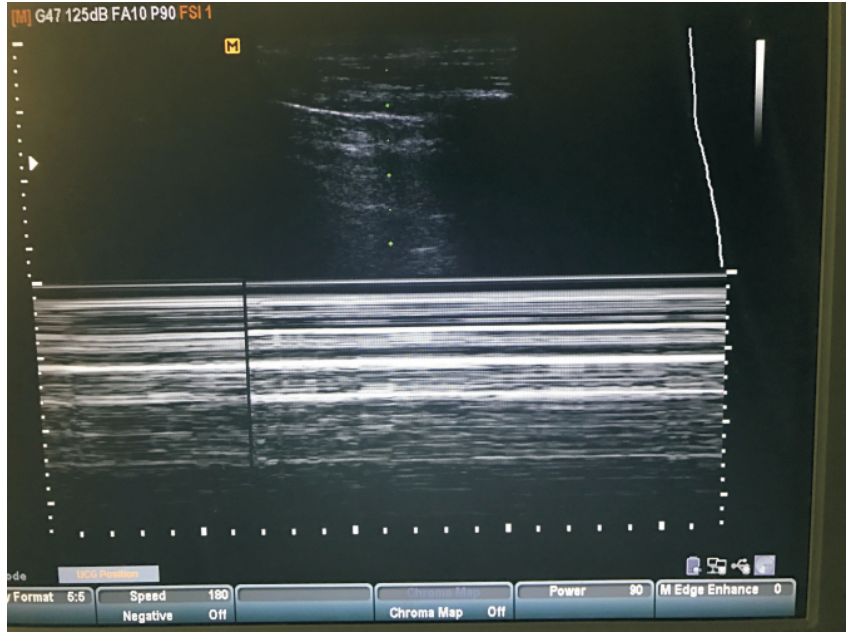

\section{Case 2}

Forty-six years old female, ASA 2, diagnosed as nonfunctioning kidney due to upper ureteric stone with hypertension taking amlodipine $5 \mathrm{mg}$, was scheduled for open nephrectomy. Pre-anesthetic check up was done, written consent was taken and she was premeditated with diazepam $5 \mathrm{mg}$ day prior to surgery. On day of surgery standard monitoring ECG, SPO2, BP was attached with intravenous line secured on left dorsum of hand. General anesthesia was induced with $2 \mathrm{mg}$ midazolam, 100 micrograms of fentanyl $90 \mathrm{mg}$ propofol and $6 \mathrm{mg}$ vecuronium and trachea was intubated. Patient was kept in left lateral position during surgery. Anesthesia was maintained with isofurane, vecuronium and fentanyl. During surgery twelfth rib was resected but at the end of surgery small pleural opening was noticed and repaired as well. Breath holding maneuver was done at end of inspiration, no air bubbles were apparent. Lung scan (My sono, Medison), using linear 
probe, was done to reconfirm pneumothorax. The scan showed presence of A-line (figure 3), sliding and seashore signs in B-mode, no barcode sign and lung point in M-mode. Pneumothorax was ruled out. Muscle relaxant was reversed with $2.5 \mathrm{mg}$ neostigmine and $0.6 \mathrm{mg}$ glycopyrolate and trachea was extubated. Patient regained consciousness, oxygen saturation was maintained and patient shifted to recovery room.

Figure 3 :

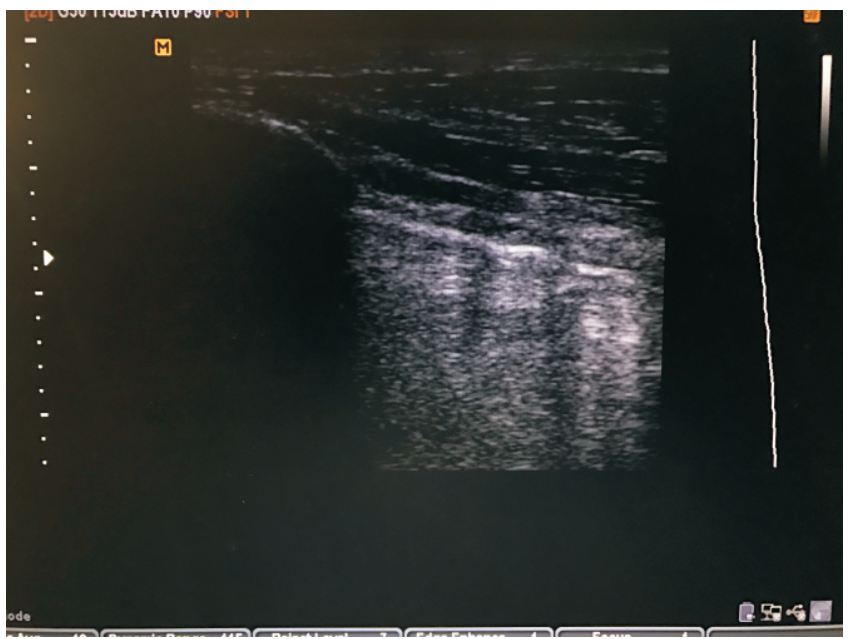

\section{DISCUSSION}

Lung scan can be useful diagnostic tool for anesthesiologist in operative room. ${ }^{2}$ As discussed, in the first case, pneumothorax was suspected, thus confirmed by ultrasound, prompt management was initiated however in the second case pneumothorax was ruled out hence the insertion of chest tube was avoided. Transfer of both patient to radiological suite for chest $\mathrm{X}$-ray was evaded. Hence lung scan can be useful in either ways as to initiate proper management or to avoid unnecessary interventions.
Moreover lung scan could be useful in various aspects of diagnosis for early detection of iatrogenic pneumothorax during various procedure like central venous catheterization, paravertebral block as Jin Suk Park et al had used ultrasound to aspirate intraplural air of the pneumothorax caused by cervical epidural and unilateral paravertebral block $^{3}$

Detecting pneumothorax by lung scan does not require high level of skill rather can be interpreted by understanding few ultrasonography terms like lines, modes, signs and point. B-mode is known as brightness mode, which is corresponded with the amplitude of reflected sound waves by the tissue while M-mode is known as motion mode, which is corresponded with waves determining the motion of underlying tissue. The terms associated with normal lung scans are lung sliding, A-lines and bat sign detected in B-mode, seashore sign in M-mode and pleural line in both B-and M-mode. Similarly terms associated with pneumothorax include absence of lung sliding in B-mode, the loss ofcomet-tail artifacts or B-lines, presence of barcode sign instead of seashore sign and visualization of lung point in M-mode. ${ }^{1}$

\section{CONCLUSION}

Lung scan not only detects pneumothorax but also helps anesthesiologist for the diagnosis of pleural effusion, consolidation, pulmonary edema and acute respiratory distress syndrome (ARDS). Bedside lung ultrasound in emergency (BLUE) protocol and fluid administrated by lung sonography (FALL) protocol are recently being recommended to be used in emergency department and ICU settings. ${ }^{4}$ Thus, anesthesiologist should be encouraged to learn lung scan as it obviously aids as added diagnostic tool for day-to-day practice for early diagnosis of lung pathologies and better patient care.

\section{REFERENCES:}

1. Laban F Husain, Laura Hagopian, Derek Wayman, William E Baker, and Kristin A Carmody. Sonographic diagnosis of pneumothorax. J Emerg Trauma Shock. 2012 Jan-Mar; 5(1): 76-81

2. Demicha Rankin, Paul S. Mathew, Lakshmi N. Kurnutala, Suren Soghomonyan, Sergio D. Bergese. Tension Pneumothorax During Surgery for Thoracic Spine Stabilization in Prone Position: A Case Report and Review of Literature. Journal of Investigative Medicine High Impact Case Reports'. 2014 apirl-june 2(2): 2324709614537233

3. Jin Suk Park, MD, Young Hoon Kim, MD, Su Ah Jeong, MD, and Dong Eon Moon. Ultrasound-guided Aspiration of the latrogenic Pneumothorax Caused by Paravertebral Block -A Case Report. Korean J Pain. 2012 Jan; 25(1): 33-37.

4. Lichtenstein DA. BLUE-protocol and FALLS-protocol: two applications of lung ultrasound in the critically ill. Chest. 2015 Jun; 147(6): 1659-70. 